\title{
TRANSVERSE INSTABILITIES
}

\author{
X. Buffat, D. Amorim, S. Antipov, G. Arduini, N. Biancacci, L. Carver, S.V. Furuseth, \\ G. Iadarola, K. Li, L. Mether, E. Métral, N. Mounet, A. Oeftiger, A. Romano, \\ G. Rumolo, B. Salvant, M. Schenk, CERN, Geneva, Switzerland \\ T. Pieloni, C. Tambasco, EPFL, Lausanne, Switzerland
}

\begin{abstract}
The observations of coherent instabilities in all operational phases of the LHC during Run 2 are summarised, describing their impact on the performance. The evolution of the mitigation strategies and of the beam instability models since the start up of the LHC are described and serve as a basis for the development of a strategy for Run 3. An emphasis will be put on the new diagnostics and tools implemented for the understanding of the instability mechanisms that affected the operation, as well as future needs.
\end{abstract}

\section{INJECTION}

At injection the beam stability remained dominated by the effect of electron clouds, even after scrubbing. To counteract the corresponding instability, a high chromaticity, damper gain and amplitude detuning is required [1]. Consistently, these stabilising measures can be relaxed when operating with single bunches as well as with $8 \mathrm{~b} 4 \mathrm{e}$ or $50 \mathrm{~ns}$ bunch trains featuring reduced electron cloud build up. In 2018, a weak blow up of the bunches at the end of $25 \mathrm{~ns}$ bunch trains was observed sporadically in the vertical plane of both beams, an illustration of this instability is shown in Fig. 1. Not only the blow up is small enough to be barely visible at top energy, once the other bunches have also blown up significantly [2], but also the fraction of bunches affected is marginal, as can be viewed from the corresponding statistics in Fig. 2. The stability margin was not explored, since the beam degradation resulting from the stabilising measures might have reduced the machine performance. Nevertheless, it was observed that this instability is worsened by linear coupling with a reduced tune separation [1]. The necessary control was obtained with a systematic measurement and correction using the ADT-AC dipole since $2017[3,4]$ and an automatic Laslett tune shift compensation [5]. The weaker instabilities observed in 2018 with a well corrected machine might also have existed in 2015 and 2016, however the absence of bunch-by-bunch oscillation measurement (the Transverse Damper (ADT) activity monitor was introduced mid-2017) and the slow scanning Synchrotron Radiation Telescope (BSRT) did not allow the measurement of such small effects. In 2017, few signs of this instability in the first part of the year were observed, thanks to the introduction of a fast scanning BSRT, allowing for multiple emittance measurements per bunch in a single injection plateau. However, the $8 \mathrm{~b} 4 \mathrm{e}$ scheme was used for most of the year as a mitigation for 16L2 events [6]. During this phase, no instabilities were observed neither in the fast scanning BSRT nor the ADT activity monitor.

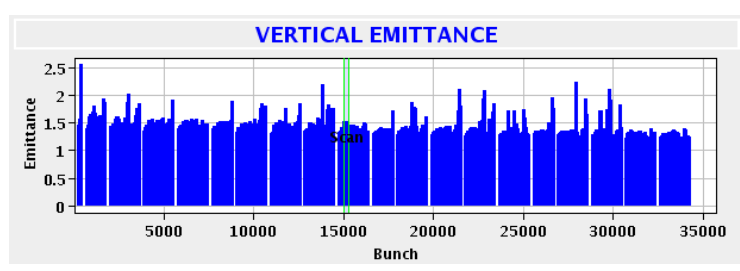

Figure 1: Bunch by bunch emittance as shown by the BSRT display at the end of the injection plateau during which weak electron cloud instabilities affected the emittance of bunches at the end of the trains.

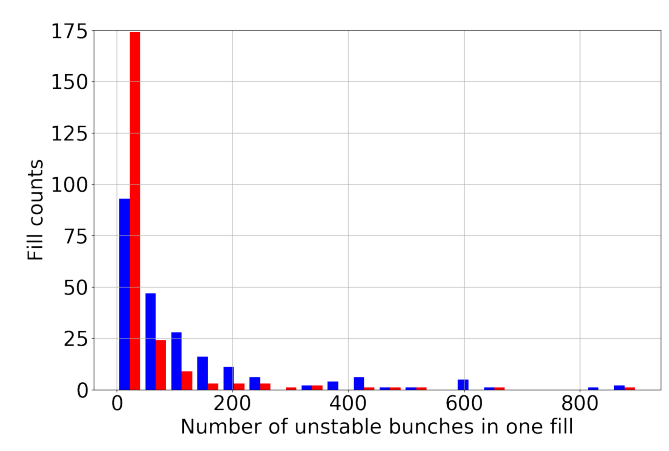

Figure 2: Histogram of the number of bunches per fill that were affected by weak electron cloud instabilities with $25 \mathrm{~ns}$ bunch trains over the year 2018 for beam 1 and 2 in blue and red respectively.

An instability with similar features was observed in the horizontal plane only with $25 \mathrm{~ns}$ beams. This instability was triggered by the leakage of the transverse excitation for injection cleaning on the circulating beam. To mitigate this instability [7], the injection cleaning was used only when needed. Due to its slow growth rate and the complexity of the interaction between the beam and the electron clouds, this instability is presently poorly known, detailed numerical studies are planned. Nevertheless, two important experimental observations can be mentioned. First, the reduction of the RF voltage from $6 \mathrm{MV}$ to $4 \mathrm{MV}$ in August $2018 \mathrm{did}$ not have a significant impact on the injection instabilities. There are signs that the instability could be dependent on the bunch length in B2, however the observables are not significant enough to draw conclusions [8]. Secondly, a Machine Development session (MD) was performed with both low $\left(7 \cdot 10^{10} \mathrm{p} / \mathrm{bunch}\right)$ and high intensity $\left(2 \cdot 10^{11} \mathrm{p} / \mathrm{bunch}\right)$ $12 \mathrm{~b}$ trains, showing that the high intensity trains remained 


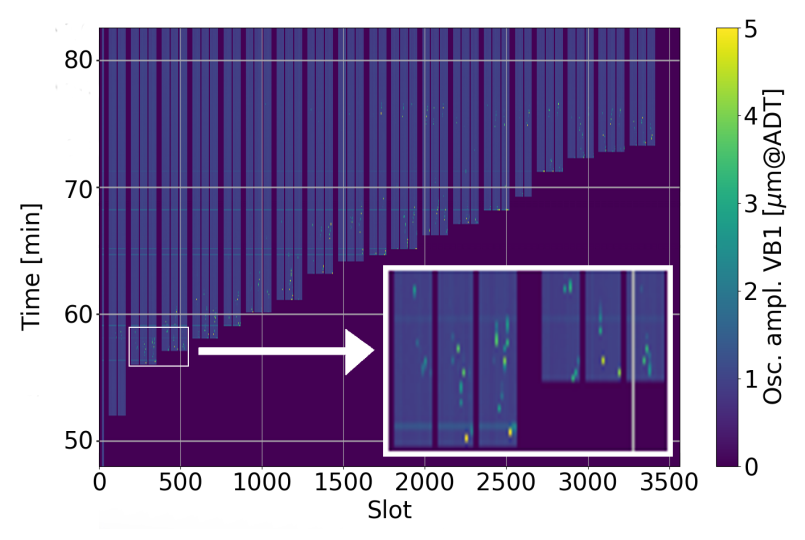

Figure 3: Bunch by bunch oscillation amplitude measured by the ADT activity monitor during injection and the beginning of the ramp (fill 7037). Transverse instabilities are observed in the few minutes following the injection of the bunch trains, as well as later on once the full beam has been injected, i.e. during the beam mode PRERAMP and beginning of RAMP.

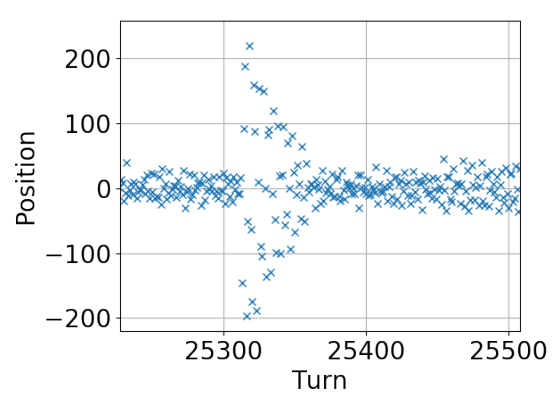

Figure 4: Turn-by-turn position of bunch 577 of B2 in the vertical plane measured by the ADTObsBox 3 minutes after the declaration of the beam mode RAMP but prior to the start of the power converter functions in fill 6988.

stable through the whole injection process with the octupoles turned off, while the low intensity trains could not be stabilised with a high strength [9]. This favourable scaling of the beam stability with high bunch intensity is expected due to a reduction of the central electron density in the dipoles [10] and a reduction of the electron cloud build up in the quadrupoles [11]. Currently no further mitigations are foreseen for this instability. We note that a deeper understanding of the mechanism would require further diagnostics on the leakage to the circulating beam.

\section{RAMP}

Until 2018, no signs of coherent motion could be observed during the ramp. In particular, the reproducibility and regularity of the blow up observed during the ramp [2] do not seem compatible with the chaotic nature of the self stabilisation mechanism leading to emittance growth or beam losses during a coherent instability. The absence of instability was confirmed with the introduction of the ADT activity monitor, when operating with the 8b4e scheme in 2017 [7]. In 2018, however, some activity was observed mainly in the vertical plane of both beams and affecting bunches at the end of the trains (Fig. 3). Since these instabilities were observed within the first two minutes of the ramp, i.e. below $600 \mathrm{GeV}$, it is likely that they are of the same nature as those observed during the injection plateau.

The detailed analysis of the ADT activity monitor and ADTObsBox data during the ramp also revealed in three cases coherent oscillations affecting few tens of consecutive bunches for about 50 turns (Fig. 4). The rise time of a few turns is not compatible with known collective instability mechanism, but could be the result of an external excitation. No significant impact could be observed on the quality of those bunches, in terms of transverse emittances and intensity. The cause of theses oscillations is unknown and would likely require further diagnostics, e.g. internal to the excitation module of the ADT in order to be understood.

\section{TOP ENERGY}

In this section we start by summarising the main issues encountered in Run 1, then discuss the various improvements to the instability model that were developed, allowing eventually for a discussion of the operation during Run 2.

\section{Operation in Run 1}

From the point of view of beam instabilities, the machine was operated in two distinct regimes in 2012. During the first part of the year, the chromaticity was set at 2 units, as specified by the LHC design [12], and the octupole polarity chosen such that the direct amplitude detuning term is negative, referred to as the negative polarity, in order to obtain the largest stability diagram for a given current, assuming a Gaussian beam distribution and neglecting two-beam effects [13]. In these conditions, strong instabilities leading to beam losses and eventually dumps when bringing the beams into collision appeared sporadically [14]. While these events remained rare for the first weeks of high intensity operation, their rate increased suddenly after the second technical stop, resulting in an unacceptable reduction of the machine performance [15]. To mitigate this instability, the chromaticity was increased to 7 then 15 units and the polarity of the octupole reversed within a few fills [16]. As a result, a weaker instability appeared at the end of the squeeze, with a marginal impact on the machine performance but nevertheless could not be cured. At the end of Run 1, many questions remained unanswered on the instabilities in the two regimes of operation of the last year, making projections for the Run 2 difficult [17]. In particular, the need for the full strength of the octupoles to maintain the beam stability at an energy of 4 $\mathrm{TeV}$ suggests that strength would be lacking significantly at 6.5 TeV due to the increased beam rigidity and the adiabatic damping of the transverse emittances, leading to a reduction of the tune spread, and consequently Landau damping while the collimator gaps, i.e. the impedance, would remain 
similar. This motivated a strong effort in the development of instability models as well as new diagnostics which are discussed here and in the next section respectively.

\section{Instability model}

In order to obtain an accurate description of the beam stability, several models were developed. In particular, the introduction of the transverse damper in the framework used to compute the coherent tune shift driven by the impedance (i.e. Vlasov perturbation theory) $[18,19]$ revealed that the damper affects not only the 0 -mode but also higher order head-tail modes. Indeed, the latter acquire a dipole component either due to the chromaticity or due to the impedance itself making them detectable by the damper, leading to damping as well as destabilisation in some cases [20]. The comparison of the coherent tune shifts from this theory with the stability diagram obtained introducing the tune spread [21] is considered as the most accurate model for the beam stability at the moment, it could be verified with macro-particle tracking simulations at several occasions [19, 22-24]. Yet, this method is based on strong assumptions on the nature of the coherent modes, that can be questioned in the presence of a strong impedance and/or a strong damper. Models relaxing such assumptions are currently under development [25].

Amplitude detuning Already within the standard instability model an accurate knowledge of the amplitude detuning is crucial to evaluate the stability diagram. The inclusion of the effect of long-range, offset and head-on beam-beam interactions [26] as well as linear and non-linear magnetic errors, in particular in the triplets [27], on the amplitude detuning, i.e. on Landau damping, explained several instabilities. Most importantly, the errors generating (non)linear coupling, were found to have a significant impact on the amplitude detuning through the mechanism of amplitude dependent closest tune approach [28], resulting in loss of Landau damping [23].

Transverse beam distribution Along with the amplitude detuning, the beam distribution plays an important role in Landau damping. Not only the impact of the tail has a strong impact [13], diffusion mechanism may also lead to modifications of the 4D transverse distribution in a way that would be inaccessible with profile measurements, yet have a strong impact on the beam stability [29]. Diffusion driven by the excitation of resonance by non-linear forces, such as beam-beam interactions could lead to such an effect, however detailed simulations studies suggest that this is not sufficient to explain the instabilities observed in the second part of 2012, even though dynamic aperture wise, this configuration was much more critical than any of the ones reached in Run 2 [30]. On the other hand, the detrimental impact on the beam stability of an external source of noise could be demonstrated experimentally in 2018. The main feature of this instability is to exhibit a latency, currently interpreted as a slow modification of the beam distribution under the influence of both the external noise and the coherent forces, e.g. the wake fields, resulting from the forced beam oscillation [31, 32]. Also, it was observed that the harmonic transverse excitation introduced in the frame of beam transfer function measurements also triggers instabilities, which could be reproduced in simulations [33, 34]. Accurate models describing these mechanism are developed in order to assess potential limitations with high brightness beams. In the mean time, an improvement of the machine noise floor is expected thanks to an improvement of the ADT pickup resolution [35].

Electron clouds The role of electron clouds on the beam stability at top energy is significantly reduced w.r.t. injection energy due mostly to the increased beam rigidity and the reduction of multipacting in the main quadrupoles [11]. A slight difference between the octupole current required to ensure the stability of $25 \mathrm{~ns}$ trains w.r.t. other types of beams was observed in 2017 [36] but could not be reproduced in 2018 [37]. On the other hand, a strong instability was observed during stable beam in 2016, often called the Pop-corn instability. Both its strength, overcoming Landau damping generated by the large head-on driven tune spread, and the fact that only the bunches at the end of the trains were affected pointed towards an effect of the electron clouds. This instability can be explained by a variation of the electron cloud distribution in the dipoles when the bunch intensity is reduced. Whereas the total amount of electrons, proportional to the heat load, remains approximatively constant. The density at the centre of the dipole, i.e. at the beam position, affects its stability strongly [10]. The instability was contained by increasing the chromaticity in stable beam to $\approx 22$ units. This mitigation was no longer needed in 2017 due to the operation with $8 \mathrm{~b} 4 \mathrm{e}$ beam reducing significantly the multipacting. The instability was also not observed in 2018 while the conditions were similar to those of 2016, possibly due to a conditioning of the parts of the beam screen surface involved in the build up of the central density.

Non-linear chromaticity The impact of non-linear chromaticity on the beam stability was studied in detail, in particular it was demonstrated experimentally that a sextupole powering scheme enhancing second order chromaticity could relax entirely the need for octupoles [38]. The compatibility of such a scheme with operation requires further studies in particular concerning its effect on the dynamic aperture in collision as well as machine protection aspects with the off-momentum $\beta$-beating induced. Nevertheless it has a significant potential to further enhance the beam stability.

Longitudinal beam distribution The longitudinal beam distribution affects both the interaction of the beam with the transverse wake fields as well as the transverse 
modes of oscillation due to the variation of the synchrotron tune with the longitudinal action, thus impacting the transverse stability of the beam. Following the observation of strongly distorted longitudinal distribution at flat top [39], the impact was estimated using PyHEADTAIL [40]. The nature of the most unstable mode is affected, while the resulting impact on the stability threshold remained marginal for the configurations tested, i.e. with chromaticities around 15 units.

Impedance model The beam stability depends on the combined effect of various sources of impedances, each described mainly by two complex functions of frequency, the so-called dipolar and quadrupolar impedances. Currently the impedance model is based on various electromagnetic computations and simulations using the design over the various objects and the best knowledge of the material properties [41, 42]. Some parts were validated with bench measurements while others are out of reach [43]. To cope with the remaining uncertainties and the potential non-conformities, a beam based validation of the model is therefore desirable, in particular in view of implementing efficient mitigations required to operate reliably with higher brightness after the implementation of the LHC Injector Upgrade (LIU)[44]. The measurement of the instability threshold mixes the uncertainty on the impedance model together with the uncertainties on the instability model, in particular Landau damping. Real tune shift measurements while moving the position of the collimator jaws allowed for a characterisation of the effective imaginary impedance of individual collimators [45], including the low-resistivity materials for the collimator upgrade [46]. Single bunch rise times were also used to characterise the total effective real impedance [7], however a non-destructive method would need to be devised in order to detail the model at the level of individual collimators. Studies are ongoing to understand whether there exist sources of impedance that are not properly included in the model compatible with the data collected. At the moment it seems that the observations are compatible with an uncertainty of less than $50 \%$ on the total effective impedance [45].

It is interesting to note that the nature of the instability mechanism, i.e. a high order head-tail modes driven by the impedance including the impact of the damper, is supported by the agreement between the predicted azimuthal and radial mode numbers with observations of the mode tune and head-tail oscillations pattern $[38,47,48]$. This suggests that the impedance is indeed the main driver of the instability and therefore the loss of Landau damping the main cause.

Operation with offset beams The operation with offset beams requires special attention, due to the strong non-linear behaviour of the beam-beam force in this regime, having a significant impact on the amplitude detuning. In particular, a critical point is reached at full separations between 1.5 to $2 \sigma$, whereas the stability diagram becomes very large for separations below $1 \sigma$ due to the large tune spread driven by head-on beam-beam interactions [26]. This reduction of

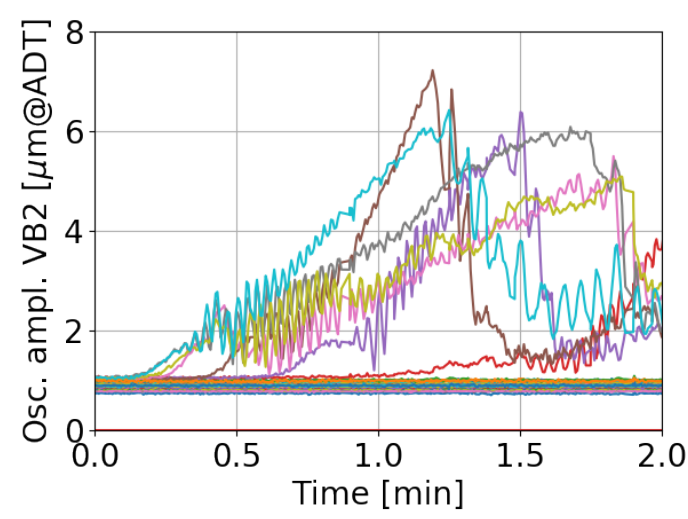

Figure 5: Oscillation amplitude of individual bunches measured by the ADT activity monitor during the round ATS optics MD [49], with a train of $48 \mathrm{~b}$ per beam colliding in IPs 1 and 5 at a crossing angle of $120 \mu \mathrm{rad}$ with $-300 \mathrm{~A}$ in the octupoles and a telescopic index of 3.1. In this experiment, PACMAN bunches are expected to be most critical [50], they became unstable at a full separation of $1.6 \sigma$ as expected. The time axis starts when this separation was reached.

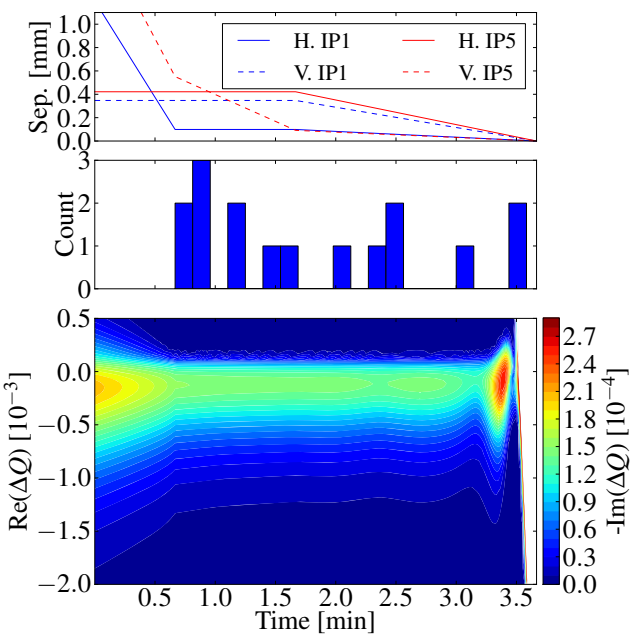

Figure 6: Illustration of the slow collapse of the separation bumps implemented in the first part of 2012 (top plot), leading to a significant amount of time spent in a configuration where the stability diagram is reduced (bottom plot). The statistics of the instabilities observed when operating with the negative polarity of the octupoles show that most of the instabilities occurred during this quasi-steady phase. Such a slow collapse of the separation bumps was avoided in Run 2. 
Landau damping is a problem mainly when the machine is meant to stay steadily in a configuration with offset beams, e.g. luminosity levelling by more than $\approx 20 \%$ with a transverse offset. The positive polarity is usually favourable in this condition, as confirmed in MD [49], but can remain critical. For example, instabilities were observed with the positive octupole polarity during IP2 and IP8 VdM scans in 2017 [7]. A robust solution for such instabilities is to ensure that every bunch experiences a least one head-on collision in one of the IPs at all times, in case this cannot be achieved, e.g. for special test, the octupole requirement has to be reviewed as they might differ significantly w.r.t. optimal settings for regular operation.

In the present operational scheme, the minimum of stability is crossed rapidly when the beams are brought into collision, preventing the development of slow instabilities in this phase, similarly to the crossing of transition in lower energy machines. This was confirmed in MD [49] by performing a fast and slow scan of the separation between the beams in IPs 1 and 5 starting at $8 \sigma$. As shown in Fig. 5, an instability was observed only during the slow scan, within few tens of seconds spent steadily at the critical separation. One may note that the instabilities observed resulted in emittance growth without losses indicating that those instabilities are of different nature w.r.t. the ones causing large losses and eventually a beam dump in the first part of 2012. Two main differences between this experiment and the instabilities in 2012 may explain this change of behaviour. First, the chromaticity was $\approx 0$ in the latter, whereas $\approx 10$ units in the first. Secondly, the beam dumps of 2012 occurred in a configuration where the beams were separated by 6 to $10 \sigma$ during a collapse of the separation bump slower than achievable by the corresponding kickers, as illustrated in Fig. 6 . In this configuration the stability diagram was reduced during the collapse of the separation bumps due to the interplay with octupoles with the negative polarity, long-range and offset beam-beam interactions on the tune spread. However it remained about a factor 3 above the stability threshold, suggesting that other effects such as those mentioned above played an important role in this instability. The fact that, in the same MD [49], no instabilities have been observed with beams separated at $8 \sigma$ for various octupole currents suggest that we have obtained a sufficient control on the relevant parameters to prevent the instability.

\section{Operation in Run 2}

The octupole and chromaticity settings of the end of 2012 were used to start with after the LS1. While the chromaticity was kept high over Run 2, the octupole current could be reduced to levels compatible with an increase of the beam brightness over the years as shown by Fig. 7. The instability observed at the end of the squeeze in 2012 was no longer observed in 2015. With present models, this can be understood as a modification of the effect of the triplet non-linearities as well as linear coupling, two critical ingredients for the beam stability that were at the time uncontrolled. In fact the first attempts to reduce the octupole in 2016 were stopped by in-

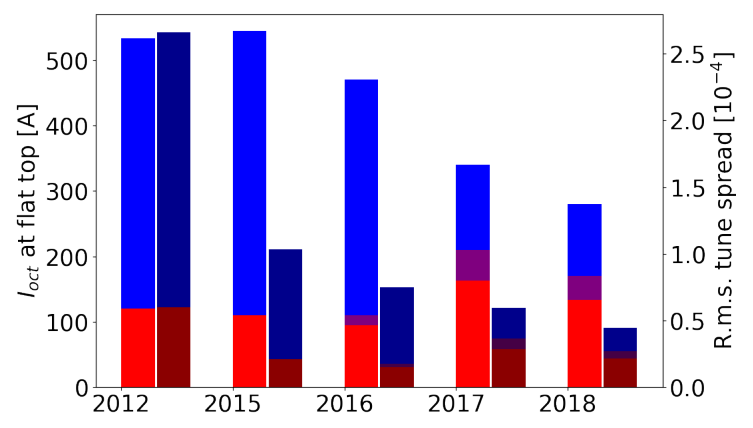

Figure 7: In light blue, the octupole current at the end of the ramp used regularly for physics operation in the standard condition of the corresponding year. In 2012, the second half of the year is considered, i.e. with the positive octupole polarity and a high chromaticity, comparable to the Run 2. In 2017, the operation with nominal $8 \mathrm{~b} 4 \mathrm{e}$ beams is considered. In light red, the corresponding prediction of the instability model. The shaded red area corresponds to the equivalent octupole current that is generated by longrange beam-beam interaction for the most critical bunches, i.e. PACMAN bunches. The darker bars represents the same values translated in r.m.s. tune spread taking into account the beam energy and transverse emittances.

stabilities during the execution of orbit manipulations around IP5 (in ADJUST) aiming at maximising the acceptance of the forward experiment TOTEM [51]. It is likely that the orbit changes have led to variations of the amplitude detuning generated by IR non-linearities as well as feed down to linear coupling, leading to a loss of Landau damping [52]. Let us note that the octupole requirement at the end of the ramp has decreased in 2016 w.r.t. 2015 due to the introduction of the squeeze during the ramp, introducing a favourable tune spread driven by long-range beam-beam interaction already at the end of the ramp. In 2017, the introduction of tight collimator settings [53] increased significantly the impedance and thus the required octupole current. Yet at the same time, the octupole current could be reduced for physics operation. This large improvement could be attributed on one side to the introduction of IR non-linear corrections [54] thus removing contributions to the tune spread equivalent to about $150 \mathrm{~A}$ in the octupoles at $\beta^{*}=0.4$ and most importantly coupling variations due to orbit variations via feed-down effects. On the other side, the online linear coupling measurement and correction tool $[3,4]$ avoided drifts that reached more than $4 \cdot 10^{-3}$ in 2016 [55], thus reducing significantly Landau damping when operating with collision tunes featuring a separation of $10^{-2}$ as of the tune change performed before the squeeze. In 2017, the reduction of the octupole current was artificially stopped by 16L2 events [56], whereas in 2018 the octupole current used for operation could be reduced to a factor 2 higher than the predictions, in agreement with the value obtained in dedicated experiments (Fig. 8a). In 2018, 


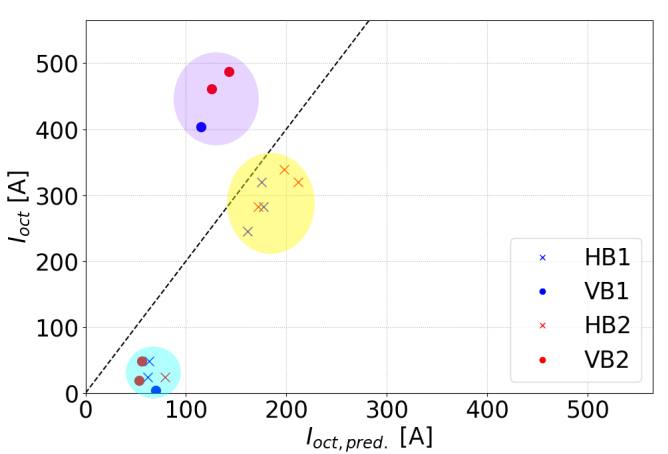

(a) Octupole scans

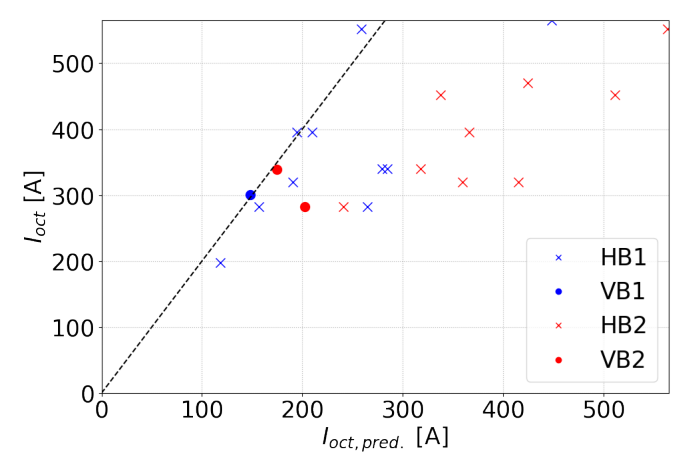

(b) Instabilities in controlled conditions

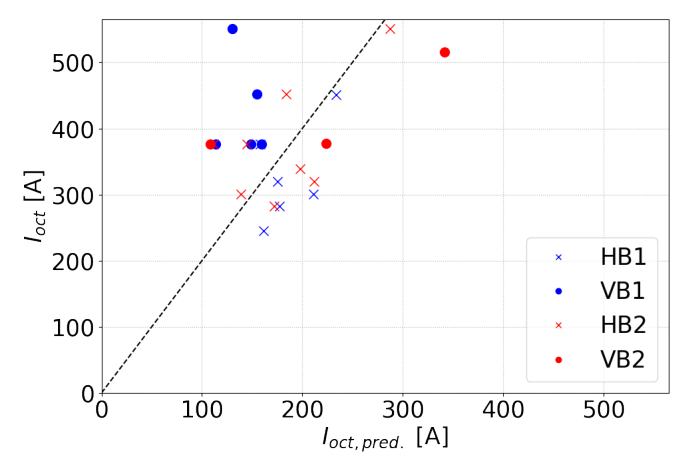

(c) Instabilities in uncontrolled conditions

Figure 8: Comparison of predicted instability threshold expressed in octupole current, compared to the octupole current at the moment of the instability. The upper plot shows the results of three octupole scans, with $\approx 10$ minutes spent at each step for three different configurations highlighted in yellow, purple, cyan corresponding respectively to the operational configuration (positive octupole polarity, high damper gain), the same configuration but without gain and an MD configuration with standard damper settings, a highly telescopic optics $\left(r_{A T S}=3.1\right)$ and the negative octupole polarity. The middle plot shows all other instabilities with standard damper settings and for which an effect of linear coupling, Pop Corn-like effects or reduction of Landau damping due to offset collisions can be excluded. The lower plot show all instabilities that were excluded. The factor 2 between predicted and empirical stability threshold is marked with black dashed line. it was not attempted to reduce the current further for regular operation.

In the configuration that has become standard since the second part of 2012 , i.e. with the positive polarity of the octupoles and a chromaticity around 15 , the measurements performed by reducing the octupoles in short steps of $\approx 1$ minutes lead to results compatible with the model $[1,51]$, whereas experiments with longer steps $(\approx 10$ minutes $)$ revealed a difference of about a factor 2 [7]. As discussed previously, this observation of an instability latency is compatible with a beam dynamics model including the effect of noise. Nevertheless, the accuracy of this model was, and still is limited. Therefore, for operation in Run 2 as well as MDs it was recommanded to keep the margins associated with this empirical factor [7]. We should note that currently this empirical factor cannot easily be transposed to other configurations (reduced damper gain, negative octupole polarity (Fig. 8a), reduced chromaticity [47]) justifying further studies dedicated to its understanding.

The other instabilities, which do not result from a voluntary reduction of the octupole current to measure the threshold, are shown in Figs. 8b and 8c. The latter shows instabilities that could be linked to the presence of uncorrected linear coupling, offset beam-beam collision or unusual damper settings, whereas the middle plot shows all instabilities that occurred in conditions for which the relevant parameters are under control. These plots illustrate the empirical factor required in controlled conditions as well as the large increase of the required octupole current in uncontrolled conditions. The main cause for the instabilities that occurred below the empirical threshold in controlled conditions is the injection of unexpectedly bright beams in special tests, i.e. mostly MDs. While it may seem natural that bunch trains are more critical from the point of view of beam stability, the stability threshold is in fact dominated by the single bunch brightness, even with a large number of bunches. Since many of the beam types available from the injectors, in particular the single bunches, are brighter than the physics beams, their stability is more critical and the octupole current needs to be adjusted accordingly. Additionally, the presence of longrange beam-beam interactions when operating with trains is favourable for the beam stability and reduces the octupole requirement. This additional tune spread needs to be compensated when operating in conditions without long-range interactions.

\section{First look at Run 3}

The operation of the LHC with less than half of the available current in the octupole at the end of Run 2 suggests that the increase of the beam brightness by a factor 2 thanks to the implementation of the LIU is within reach. Nevertheless we note that the favourable contribution of long-range beam-beam interactions is included in these considerations, therefore the operation with non-colliding bunches for commissioning, intensity ramp up or dedicated tests would be prohibited in these conditions. Moreover, additional octupole strength will be needed to increase the energy from 


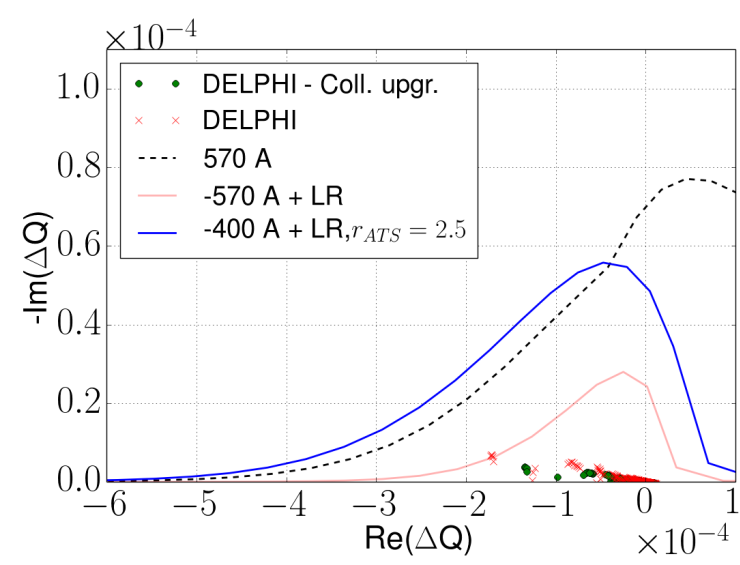

Figure 9: Comparison of the stability diagrams and the coherent tune shifts at the end of the ramp for the Run 3 configuration [57] with an intensity of $1.8 \cdot 10^{11} \mathrm{p} / \mathrm{bunch}$ and a transverse emittance of $1.8 \mu \mathrm{m}$. For reference, the red crosses mark the coherent tune shifts in absence of low impedance collimator upgrade [58]. The stability diagram with the positive polarity of the octupoles and without beambeam interaction is shown in dashed. With the negative polarity, the detrimental impact of the long-range interactions at the end of the ramp (red curve) needs to be compensated by boosting the octupole effectiveness introducing a (anti-)telescopic index $r_{A T S}$ (blue curve).

6.5 to $7 \mathrm{TeV}$, as well as to ensure the stability during the collapse of the separation bumps, as Landau damping is not linear with the octupole strength in this phase[59]. The first phase of the low impedance collimator upgrade addresses these issues by reducing the required tune spread [60] as illustrated in Fig. 9. It has to be noted that this configuration does not leave any margin for other uncontrolled sources of tune spread and therefore imposes significant constraints on the optics correction, linear and non-linear. The robustness of the operation against parameter drifts as well as potential IR non-linear corrector failure can be significantly improved by increasing the effective strength of the arc octupoles with the ATS optics early in the cycle, i.e. during the ramp. Additionally, such a scheme allows to maintain the required tune spread if the octupoles and the long-range beam-beam interactions interfere unfavourably for Landau damping, i.e. when operating with the negative polarity of the octupoles (Fig. 9). This opens the possibility to optimise the performance of the machine using the favourable interplay between the long-range beam-beam interaction and the octupole on the dynamic aperture in this configuration.

The high potential of this scheme was tested in MDs [49], with a telescopic index of 3.1. The reduction of octupole current required to maintain stability of a single beam was measured beyond expectations, as shown in Fig. 8a. The threshold measured lower than expectations in this configuration with negative polarity of the octupole may be attributed to a large tail population [13] which is not measurable with existing diagnostics.

One may note that the extrapolation of the margin linked to the empirical factor between predicted and observed stability threshold to higher beam intensities suffers large uncertainties, nevertheless the current experience with higher bunch intensities is rather positive. In the frame of MDs as well as high pile-up tests, single bunches of brightness exceeding the HL-LHC target have been brought into collision without instabilities, even with tight collimator settings [61], as well as $12 \mathrm{~b}$ trains exceeding the intensity anticipated for run III [37].

\section{DIAGNOSTICS}

After Run 1, large efforts were put to improve diagnostics and machine control to both understand and mitigate instabilities, contributing significantly to the achievements described above. Here we described briefly what was provided and how they were used.

\section{Timing}

An accurate measurement of the time at which instabilities occurred is critical to understand the machine and beam configuration at the moment of the instability as well as for correlation with machine changes. For these purposes, the required precision is in the order of the timescale of the power converter, i.e. from few seconds to a minute. Moreover, since most of the instabilities observed affected one or few bunches, bunch-by-bunch measurement is critical. For example, the signal of single bunch instabilities is usually lost in the oscillation amplitude measured by the BBQ when operating with bunch trains [62]. The bunch intensity measured by the FBCT was used instead already in Run 1 . However for several instabilities self-stabilisation tends to occur with emittance growth but without losses. The bunchby-bunch emittance measurement by the BSRT was too slow to be used for such a purpose until 2017. The introduction of digital cameras allowed for a significant increase of the acquisition speed, resulting in bunch-by-bunch measurement of the transverse emittance with a time resolution of about a minute [63]. In summer 2017, the ADT activity monitor was introduced, providing bunch-by-bunch oscillation amplitude with a sub-second resolution thus becoming the main tool for instability detection [64, 65].

\section{Characteristics}

The determination of the instability type (e.g. single bunch weak head-tail, coupled bunch, mode-coupling, beam-beam, electron cloud), as well as its characteristics (tune shift, growth rate, mode number) play an important role in the understanding of the cause and the mitigation of an instability. The main observables are the bunch-by-bunch oscillation pattern, the intra-bunch oscillation pattern, the time evolution of the oscillation and its spectrum. The reconstruction of the multi- and intra-bunch mode pattern as well as the corresponding spectrum requires data for a large amount of consecutive turns, prohibiting continuous 
acquisitions. For this purpose the LHC Instability Trigger Network (LIST) was introduced in Run 2, allowing for multiple devices to send triggers when an instability is detected and acquire data for a limited amount of time when triggered [48]. The main usage of this scheme is the acquisition of the head-tail monitor [48] which acquisition was limited to 12 consecutive turns for most of Run 2 (120 turns were available towards the end of 2018) and therefore relied on such a fast triggering system. Despite the large amount of false positive, the triggers sent by the BBQ allowed for a large amount of relevant measurements.

While in Run 1 the bunch-by-bunch oscillation could be recorded for 73 consecutive turns upon a manual trigger, the introduction of the ADTObsBox in Run 2 improved significantly the situation [48, 66]. Since 2016, the ADTObsBox is connected to the LIST and provides triggers based on single bunch oscillation amplitude. The recording of a general purpose buffer was enabled by the instability triggers, thus allowing for offline analysis of 64 thousands consecutive turns of bunch-by-bunch data. These represents the only access to coupled bunch information as well as single bunch spectrum. The time evolution, most importantly the instability growth rate, can also be obtained from such data, nevertheless the ADT activity monitor is usually sufficient for that purpose and does not rely on the trigger.

The analysis of the 16L2 events thanks to post mortem acquisition of both the head-tail monitor and the ADTObsBox represents a striking example of the importance of these devices [6].

\section{Probing the models}

The determination of the timing and the characteristics of the instabilities described above were used not only to diagnose and mitigate the instabilities encountered in operation, but were also critical for several tests dedicated to the study of instabilities. We describe here other techniques that were developed to probe the various models.

Beam transfer function The beam transfer function provides a direct measurement of the stability diagram by exciting the beam with a sweeping harmonic excitation and measuring its response [21]. This tool was introduced mainly to study the modification of the beam distribution driven by noise or by non-linear diffusion mechanism that are inaccessible by transverse profile measurements and yet have a significant impact on Landau damping [67]. The resolution required for such measurements could not be reached with the present system. Moreover, the reach of this system was strongly limited by the requirement from the BBQ to operate with a single bunch in the beam under study, preventing comparison between bunches in different configurations (with/without beam-beam interactions, PACMAN effects) and preventing any trial and error in the setting up at top energy due to the long turn around. Nevertheless the stability diagram generated by the octupole could be measured at injection, also in the presence of linear coupling [67]. The implementation of a single bunch BTF measurement using the gated BBQ in 2018 significantly improved the situation and allowed for direct measurement of the interplay of beambeam interactions and the octupoles on Landau damping at top energy [68].

Controllable impedance An alternative to beam transfer function measurements was tested in 2018 using the ADT as a controllable source of impedance, introducing both an amplification term (or a negative damping gain) as well as a reactive component. This flexible impedance allows to probe the complex plane representing coherent tune shifts, thus drawing the stability diagram by observing the stability of the beam in various conditions. The proof of principle experiment at injection energy provided encouraging results [69].

Controllable noise source While the modification of the distribution and the resulting modification of the stability diagram postulated as the cause for the instability with a latency [29] could not be observed directly through beam transfer function measurements, the effect of the noise on the beam stability was confirmed using the ADT to inject noise of various amplitudes on different bunch simultaneously [32].

Single bunch kick Single bunch kicks for accurate tune measurements have been the working horse for beam-based impedance measurement. The synchronisation of the kicks with the acquisition of a dedicated buffer of the ADTObsBox allowed for efficient online and offline analysis of large amount of measurements, thus overcoming the limitation of the machine tune jitter which is in the same order of magnitude as the tune shift expected from a single collimator $\left(<10^{-4}\right)$.

\section{Machine control}

As discussed in the previous section, the introduction of an online measurement and correction of the linear coupling based on ADT-AC dipole on single bunches brought a significant improvement of the octupole current required to operate reliably. The accuracy of this method is currently limited by the accuracy of the tune measurement [70], which could be overcome using the single bunch kick capabilities that were for the moment limited to special tests. Simultaneously, the measurement of the head-tail signal during the resulting oscillations opens the possibility to measure the chromaticity on demand $[71,72]$. In particular, the capability to kick and measure a single bunch reduces the concerns over the resulting emittance growth at the design stage [12]. This example illustrate the potential of the tools developed in Run 2 as expert diagnostics for instabilities to improve the operation of the machine. 


\section{CONCLUSION}

Whereas the operation in the last year of Run 1 suffered from beam instabilities, the performance in Run 2 was never limited by beam instabilities. On the other hand the bunch intensity remained limited by 16L 2 events.

Strong efforts were put on the understanding of the mechanism involved in the instability as well as in the characterisation of the various contributors (amplitude detuning, impedance, noise). The resulting mitigations, in particular the control of the optics (linear coupling, IR non-linear correction) and the optimisation of the operational procedures (fast collapse of the separation bumps in IPs 1 and 5, constraint on the minimum tune separation for lifetime optimisation in ADJUST, octupole reduction during the squeeze) reduced the requirements to acceptable levels in view of doubling the beam brightness in Run 3 and HL-LHC, provided the empirical factor between required and predicted octupole current, remains unchanged, which is an aspect still under study.

\section{REFERENCES}

[1] L. Carver et al., 'Instabilities and beam induced heating in 2015', in Proceedings of the 2015 Evian workshop on LHC beam operation, B. Goddard and S. Dubourg, Eds., Evianles-Bains, France, 15-17 December 2015.

[2] S. Papadopoulou et al., 'What do we understand on the emittance growth?', in These proceedings.

[3] T. Persson et al., 'Optics control in 2016', in Proceedings of the 2016 Evian workshop on LHC beam operation, T. Argyropoulos, S. Dubourg and G. Trad, Eds., Evian-lesBains, France, 13-15 December 2016.

[4] A. Calia et al., 'Operational tools from 2017 to 2018', in Proceedings of the 2017 Evian workshop on LHC beam operation, B. Goddard, S. Dubourg and G. Trad, Eds., Evianles-Bains, France, Dec. 2017.

[5] M. Schaumann, M. Solfaroli and J. Wenninger, 'Tune and chromaticity : Decay and snapback', in Proceedings of the 2015 Evian workshop on LHC beam operation, B. Goddard and S. Dubourg, Eds., Evian-les-Bains, France, 15-17 December 2015.

[6] L. Mether et al., '16L2: Operation, observations and physics aspects', in Proceedings of the 2017 Evian workshop on LHC beam operation, B. Goddard, S. Dubourg and G. Trad, Eds., Evian-les-Bains, France, Dec. 2017.

[7] X. Buffat et al., 'Our understanding of transverse instabilities and mitigation tools/strategy', in Proceedings of the 2017 Evian workshop on LHC beam operation, B. Goddard, S. Dubourg and G. Trad, Eds., Evian-les-Bains, France, Dec. 2017.

[8] N. Mounet, Impact of the bunch length on the injection instabilities, Presentation at the LHC Instability Monitoring meeting, 22 Aug. 2018.

[9] G. Iadarola et al., 'Heat load measurements with high bunch intensity (trains of 12b)', CERN, Geneva, Switzerland, Tech. Rep. CERN-ACC-NOTE-2019, 2019.

[10] A. Romano, O. Boine-Frankenheim, X. Buffat, G. Iadarola and G. Rumolo, 'Electron cloud buildup driving spontaneous vertical instabilities of stored beams in the large hadron collider', Phys. Rev. Accel. Beams, vol. 21, p. 061 002, 6 Jun. 2018.

[11] G. Iadarola et al., Digesting the LIU high brightness beam: Is this an issue for HL-LHC?, Presentation at the LHC Performance Workshop 2018, 31 Jan. 2018.

[12] O. Brüning et al., Eds., LHC design report. Geneva, Switzerland: CERN, 2004, vol. 1 : The LHC Main ring.

[13] E. Métral and A. Verdier, 'Stability diagram for Landau damping with a beam collimated at an arbitrary number of sigmas', CERN, Geneva, Switzerland, Tech. Rep. CERNAB-2004-019-ABP, Feb. 2004.

[14] T. Pieloni et al., 'Stability of colliding beams at $6.5 \mathrm{TeV}$ ', in Proceedings of the 2012 Evian workshop on LHC beam operation, B. Goddard and S. Dubourg, Eds., Evian-les-Bains, France: CERN.

[15] E. Métral et al., 'Summary of the 2-day internal review of LHC pefromance limitations (linked to transverse collective effects) during run i', CERN, Geneva, Switzerland, Tech. Rep. CERN-ACC-NOTE-2014-0006, 2014.

[16] G. Arduini, (clear?) summary of the observations on instabilities, Presentation at the LHC Machine Committee, 15 Aug. 2012.

[17] N. Mounet et al., 'Beam stability with separeted beams at $6.5 \mathrm{TeV}$ ', in Proceedings of the 2012 Evian workshop on LHC beam operation, B. Goddard and S. Dubourg, Eds., Evian-les-Bains, France: CERN.

[18] A. Burov, 'Nested head-tail vlasov solver', Phys. Rev. ST Accel. Beams, vol. 17, p. 021 007, 2 Feb. 2014.

[19] N. Mounet, 'Vlasov solvers and macroparticle simulations', in Proceedings of the ICFA-Mini Workshop on Impedances and Beam Instabilities in Particle Accelerators from 18 to 22 September, 2017., G. Rumolo, S. Petracca and I. Masullo, Eds., Benevento, Italy: CERN.

[20] E. Métral, D. Amorim, S. Antipov, N. Biancacci, X. Buffat and $\mathrm{K} . \mathrm{Li}$, 'Destabilising effect of the LHC transverse damper', in Proceedings of 9th International Particle Accelerator Conference, S.Koscielniak, T. Satogata, V. Schaa and J. Thompson, Eds., Vancouver, Canada: JACoW, 15-20 June 2014 2018, pp. 3076-3079.

[21] J. Berg and F. Ruggiero, 'Landau damping with twodimensional betatron tune spread', CERN, Geneva, Switzerland, Tech. Rep. SL-96-071-AP, Dec. 1996.

[22] R. Wasef, HEADTAIL simulation studies of landau damping through octupoles in the LHC, Master thesis, Grenoble, 2011.

[23] L. R. Carver, X. Buffat, K. Li, E. Métral and M. Schenk, 'Transverse beam instabilities in the presence of linear coupling in the large hadron collider', Phys. Rev. Accel. Beams, vol. 21, p. 044 401, 4 Apr. 2018.

[24] M. Schenk, X. Buffat, K. Li and A. Maillard, 'Vlasov description of the effects of nonlinear chromaticity on transverse coherent beam instabilities', Phys. Rev. Accel. Beams, vol. 21, p. 084 402, 8 Aug. 2018.

[25] N. Mounet, Stability diagrams, damper, mode coupling and all that sort of things, Presentation at the Hadron Synchrotron Collective effects meeting, 4 Feb. 2019.

[26] X. Buffat, W. Herr, N. Mounet, T. Pieloni and S. White, 'Stability diagrams of colliding beams in the large hadron collider', Phys. Rev. ST Accel. Beams, vol. 17, p. 111 002, 11 Nov. 2014.

[27] E. Maclean et al., 'Nonlinear optics commissioning in the LHC', in Proceedings of the 2016 Evian workshop on LHC beam operation, T. Argyropoulos, S. Dubourg and G. Trad, 
Eds., Evian-les-Bains, France, 13-15 December 2016.

[28] R. Tomás, T. H. B. Persson and E. H. Maclean, 'Amplitude dependent closest tune approach', Phys. Rev. Accel. Beams, vol. 19, p. 071 003, 7 Jul. 2016.

[29] X. Buffat, 'Transverse beams stability studies at the large hadron collider', PhD thesis, EPFL, 2015.

[30] T. Pieloni et al., 'Beam-beam effects long-range and headon', in Proceedings of the 2015 Evian workshop on LHC beam operation, B. Goddard and S. Dubourg, Eds., Evianles-Bains, France, 15-17 December 2015.

[31] X. Buffat et al., Noise, latency and transverse instabilities, Presentation at the LHC Machine Committee, 11 Jul. 2018.

[32] S. Furuseth et al., 'Md3288: Instability latency with controlled noise', CERN, Geneva, Switzerland, Tech. Rep. CERN-ACC-NOTE-2019-0011, 2019.

[33] 'Md 2722: Investigation of landau damping by means of btf measurements', CERN, Geneva, Switzerland, Tech. Rep. CERN-ACC-NOTE-2018-0016, 2018.

[34] C. Tambasco et al., 'Instability threshold measurements in the presence of a controlled external excitation', CERN, Geneva, Switzerland, Tech. Rep. CERN-ACC-NOTE-2019, 2019.

[35] X. Buffat et al., 'Noise studies with new adt pickup electronics', CERN, Geneva, Switzerland, Tech. Rep. CERN-ACCNOTE-2019, 2019

[36] L. Carver et al., 'The ghost train instability (mds 2066 and 2936)', CERN, Geneva, Switzerland, Tech. Rep. CERNACC-NOTE-2018-0013, 2018.

[37] X. Buffat et al., 'Intensity dependence on the train instability threshold', CERN, Geneva, Switzerland, Tech. Rep. CERNACC-NOTE-2019, 2019.

[38] M. Schenk, X. Buffat, L. R. Carver, R. De Maria, K. Li and E Métral, 'Experimental stabilization of transverse collective instabilities in the lhc with second order chromaticity', Phys. Rev. Accel. Beams, vol. 21, p. 084 401, 8 Aug. 2018.

[39] H. Timko, P. Baudrenghien, O. Brunner, A. Butterworth, J. E. Muller and E. Shaposhnikova, 'Operational and beam dynamics aspects of the RF system in 2016', in Proceedings of the 2016 Evian workshop on LHC beam operation, B. Goddard, S. Dubourg and G. Trad, Eds., Evian-les-Bains, France, 13-15 December 2016.

[40] A. Oeftiger, LHC flat-top instabilities and landau octupole compensation, Presentation at the HL-LHC WP2 meeting, 27 Nov. 2018.

[41] N. Mounet, 'The lhc transverse coupled bunch instability', PhD thesis, EPFL, 2012

[42] http://impedance.web.cern.ch/impedance/.

[43] F. Roncarolo et al., 'Comparison between laboratory measurements, simulations, and analytical predictions of the transverse wall impedance at low frequencies', Phys. Rev. ST Accel. Beams, vol. 12, p. 084 401, 8 Aug. 2009.

[44] G. Rumolo, 'What to expect from the injectors during Run 3', in These proceedings.

[45] D. Amorim et al., Comparison of lhc impedance model predictions to beam-based measurements, Presentation at the Hadron Synchrotron Collective effects meeting, 4 Mar. 2019.

[46] N. Biancacci et al., 'Low-impedance collimators for HLLHC', in Proceedings of 9th International Particle Accelerator Conference, S.Koscielniak, T. Satogata, V. Schaa and J. Thompson, Eds., Vancouver, Canada: JACoW, 29 Apr. - 4 May 2018 2018, pp. 1794-1797.

[47] L. Carver et al., 'Currents status of instability threshold measurements in the LHC at $6.5 \mathrm{TeV}$ ', in Proceedings of 7th International Particle Accelerator Conference, C. PetitJean-Genaz, D. E. Kim, K. S. Kim, I. S. Ko, K. R. Kim and V. Schaa, Eds., Busan, South Korea: JACoW, Aug. 2016, pp. 1434-1437.

[48] T. Levens et al., 'Instability diagnostics', in Proceedings of the 2015 Evian workshop on LHC beam operation, B. Goddard and S. Dubourg, Eds., Evian-les-Bains, France, 1517 December 2015

[49] S. Fartoukh, A. Mereghetti, M. Solfaroli, J. Wenninger, X. Buffat et al., 'Round ats optics with large tele-index', CERN, Geneva, Switzerland, Tech. Rep. CERN-ACC-NOTE-2019, 2019.

[50] X. Buffat and S. Fartoukh, Instabilities with a high tele-index, Presentation at the Hadron Synchrotron Collective effects meeting, 3 Dec. 2018.

[51] L. Carver et al., 'Instabilities and beam induced heating in 2016', in Proceedings of the 2016 Evian workshop on LHC beam operation, B. Goddard, S. Dubourg and G. Trad, Eds., Evian-les-Bains, France, 13-15 December 2016.

[52] T. Persson et al., 'Optics and correction', in These proceedings.

[53] R. Bruce et al., 'LHC machine configurations in run 2', in These proceedings.

[54] E. Maclean et al., 'New optics correction approaches in 2017', in Proceedings of the 2017 Evian workshop on LHC beam operation, B. Goddard, S. Dubourg and G. Trad, Eds., Evian-les-Bains, France, Dec. 2017.

[55] E. Métral et al., 'Summary of the half-day internal review of lhc performance limitations (linked to transverse collective effects) during run ii', CERN, Geneva, Switzerland, Tech. Rep. CERN-ACC-NOTE-2014-0006, 2014.

[56] X. Buffat et al., Stability with reduced octupoles and chromaticity, Presentation at the LHC Beam Operation Committee, 27 Jun. 2017.

[57] N. Karastathis et al., 'Report from the LHC Run-III Configuration Working Group', in These proceedings.

[58] S. Antipov et al., 'Staged implementation of lowâimpedance collimation in IR7: Plans for LS2', CERN, Geneva, Switzerland, Tech. Rep. CERN-ACC-2019-0001, 2019.

[59] X. Buffat et al., 'Status of the studies on collective effects involving beam-beam interactions at the HL-LHC', CERN, Geneva, Switzerland, Tech. Rep. CERN-ACC-NOTE-20180036, 2018.

[60] N. Mounet and X. Buffat, Run iii: Impedance considerations, Presentation at the LHC Run III working group, 21 Sept. 2018.

[61] X. Buffat et al., 'Impact of the ADT on the beam quality with high brightness beams in collision (md2155)', CERN, Geneva, Switzerland, Tech. Rep. CERN-ACC-NOTE-20180005, 2018.

[62] X. Buffat et al., Observations of instabilities during stable beam, Presentation at the LHC Beam Operation Committee, 7 Jun. 2016.

[63] G. Trad et al., 'Status of the LHC emittance diagnostics', in Proceedings of the 2017 Evian workshop on LHC beam operation, B. Goddard, S. Dubourg and G. Trad, Eds., Evianles-Bains, France, Dec. 2017.

[64] G. Kotzian, M. Soderen and D. Valuch, Real time ADT transverse instability detection is operational : What do we do with it?, Presentation at the LHC Machine Committee, 28 Jun. 2017.

[65] M. Soderen, G. Kotzian, M. Ojeda-Sandonis and D. Valuch, 
'Online bunch by bunch transverse detection in LHC', in Proceedings of 8th International Particle Accelerator Conference, G. Arduini, M. Lindroos, J. Pranke, V. Schaa and M. Seidel, Eds., Copenhagen, Denmark: JACoW, 14 - 19 May 2017, pp. 397-399.

[66] M. Soderen, D. Valuch, J. Kompulla and G. Kotzian, 'ADT and ObsBox', in These proceedings.

[67] C. Tambasco, 'Beam transfer function measurements and transverse beam stability studies for the large hadron collider and its high luminosity upgrade', PhD thesis, EPFL, 2017.

[68] C. Tambasco et al., 'Md 3292: Investigation of landau damping by means of btf measurements', CERN, Geneva, Switzerland, Tech. Rep. CERN-ACC-NOTE-2019, 2018.
[69] S. Antipov et al., 'Studies of landau damping with an antidamper', CERN, Geneva, Switzerland, Tech. Rep. CERNACC-NOTE-2019, 2019.

[70] T. Persson and R. Tomás, 'Improved control of the betatron coupling in the large hadron collider', Phys. Rev. ST Accel. Beams, vol. 17, p. 051 004, 5 May 2014.

[71] D. Cocq, O. R. Jones and H. Schmickler, 'The measurement of chromaticity via a head-tail phase shift', AIP Conference Proceedings, vol. 451, no. 1, pp. 281-288, 1998.

[72] N. Mounet, Linearized Vlasov equation with Laplace transform, Presentation at the Hadron Synchrotron Collective effects meeting, 17 Dec. 2018. 